\title{
Approximation Operators Based on L-Fuzzy Set-Valued Mappings
}

\author{
Zhengjiang $\mathbf{W u}^{1}$ Xin Liu ${ }^{2}$ Shuiting $\mathbf{W u}^{1}$ Keyun Qin ${ }^{3}$ \\ ${ }^{1}$ Intelligent Control and Development Center, Southwest Jiaotong University, Chengdu, 610031, China \\ ${ }^{2}$ Mathematics College, Liaoning Normal University, Dalian, 116029, P.R.China \\ ${ }^{3}$ Department of Mathematics, Southwest Jiaotong University,Chengdu, 610031, China
}

\begin{abstract}
In this paper, the fuzziness of Gomolińska approximation space based on uncertainty mappings was introduced. It is proved that many approximation operators could be constructed by composition of basic approximation operators.
\end{abstract}

Keywords: Approximation space, Fuzzy rough set, Approximation operator

\section{Introduction}

A rough set is a set-theory-based technique to handle data with granular structures by using two sets called the rough lower approximation and the rough upper approximation to approximation object. By using this technique, knowledge hidden in information systems may be unraveled and expressed in the form of decision rules. The classical definition of a rough set was introduced by Pawlak [1] with reference to an equivalence relation (a binary relation with reflexivity, symmetry and transitivity).

From both theoretical and practical viewpoint, the equivalence relation is a very stringent condition that may limit applications of rough sets. Various extensions of the Pawlak rough set were therefore developed from an equivalence relation to a more general mathematical concept, e.g. a similarity relation (a binary relation with reflexivity and symmetry), a covering [2], or a neighborhood system from the topological space [3].

Dubois and Prade studied first the fuzzification problem of rough sets [4], [5]. Morsi and Yakout [6] studied a set of axioms on approximation operators of fuzzy sets and defined a special family of approximation operators of fuzzy sets using the T-norms and the residuation implicators. Additionally, Radzikowska and Kerre [7] gave another general method for the fuzzification of rough sets. They defined a broad family of fuzzy rough sets, each of which is determined by a triangular norm and an implicator. But the discussions of fuzzy rough set in many of article are based on [0,1]-fuzzy set rather than L-fuzzy set. Later, Radzikowska and Kerre [8] generalized the model of fuzzy rough set to L-fuzzy rough set based on residuated lattice and discuss some basic properties of approximation operators of the L-fuzzy rough set. T. Deng [9] fuzzify the general rough approximation operators and present a new approach to fuzzy rough sets through the use of techniques provide by residuated lattice.

In those L-fuzzy rough set model, approximation operators have several types of definition. In this paper, we studies the relation of those approximation operators in L-fuzzy approximation space. It is proved that those approximation operators of L-fuzzy rough set are special cases of approximation operators based on L-fuzzy set-valued mappings.

\section{Preliminaries}

A monoid is a structure $(U, \otimes, \varepsilon)$, where $U$ is a nonempty universe, $\otimes$ is a binary operator on $U$ and $\varepsilon$ is a unit element of $\otimes$, i.e. for every $x \in U, \varepsilon \otimes x=$ $x \otimes \varepsilon=x$. A monoid $(U, \otimes, \varepsilon)$ is commutative if and only if $\otimes$ is commutative. Typical example of monoid is triangle norm on $[0,1]$, where $\varepsilon=1$.

Let $(L, \leq)$ be a poset and $\otimes$ a binary operation on $L$. The residuum of $\otimes$ is a binary operator $\rightarrow$ in $L$ satisfying the following residuated condition: for all $a, b, c \in L, a \otimes b \leq c \Leftrightarrow a \leq b \rightarrow c$. The operator $\rightarrow$ is bounded if and only if for every $x \in L, 1 \rightarrow$ $x=x$.

Definition 2.1 [10] By a residuated lattice, we mean an algebra $\boldsymbol{L}=(L, \vee, \wedge, \otimes, \rightarrow, 0,1)$, such that

(1) $(L, \vee, \wedge, 0,1)$ is a bound lattice with the top element 1 and the bottom element 0 .

(2) $\otimes: L \times L \rightarrow L$ is a binary operator and satisfies for all $a, b, c \in L$,

- $a \otimes b=b \otimes a$, 
- $a \otimes(b \otimes c)=(a \otimes b) \otimes c$,

- $1 \otimes a=a$,

- $a \leq b \Rightarrow a \otimes c \leq b \otimes c$.

(3) $\rightarrow: L \times L$ is a residuum of $\otimes$, i.e. $\rightarrow$ satisfies for all $a, b, c \in L$

$$
a \otimes b \leq c \Leftrightarrow a \leq b \rightarrow c .
$$

A residuated lattice $\mathbf{L}=(L, \vee, \wedge, \otimes, \rightarrow$ $, 0,1)$ is called complete iff the underlying lattice $(L, \vee, \wedge, 0,1)$ is complete. Given a residuated lattice $\mathbf{L}$, we define the precomplement operator $\sim$ as following, for every $a \in \mathbf{L}, \sim a=a \rightarrow 0$.

Proposition 2.2 [10]-[12], Suppose $\boldsymbol{L}=(L, \vee, \wedge$, $\otimes, \rightarrow, 0,1)$ is a residuated lattice, and $\sim$ is the precomplement operator on $\boldsymbol{L}$. Then for all $a, b, c \in \boldsymbol{L}$,

(1) $a \otimes b \leq a \wedge b, a \rightarrow b \geq b$.

(2) $a \rightarrow(b \rightarrow c)=(a \otimes b) \rightarrow c$.

(3) If $a \leq b$, then $c \rightarrow a \leq c \rightarrow b$ and $b \rightarrow c \leq a \rightarrow$ $c$.

(4) $a \leq b \Leftrightarrow a \rightarrow b=1$.

(5) $a \leq b \Rightarrow \sim a \geq \sim b$.

(6) $a \leq \sim \sim a$.

(7) $a \rightarrow b \leq \sim(a \otimes \sim b)$, $a \otimes b \leq \sim(a \rightarrow \sim b)$.

(8) If $L$ is a complete lattice, then

$$
\begin{aligned}
& \left(\vee_{i \in I} a_{i}\right) \otimes b=\vee_{i \in I}\left(a_{i} \otimes b\right), \\
& a \rightarrow \wedge_{i \in I} b_{i}=\wedge_{i \in I}\left(a \rightarrow b_{i}\right), \\
& \vee_{i \in I} a_{i} \rightarrow b=\wedge_{i \in I}\left(a_{i} \rightarrow b\right), \\
& a \rightarrow \vee_{i \in I} b_{i} \geq \vee_{i \in I}\left(a \rightarrow b_{i}\right), \\
& \wedge_{i \in I} a_{i} \rightarrow b \geq \vee_{i \in I}\left(a_{i} \rightarrow b\right)
\end{aligned}
$$

Definition 2.3 [9] Let $\otimes$ be a conjunction on a complete residuated lattice $L, A$ L-fuzzy relation $R_{L}$ on $U$ is a L-fuzzy set $\left.R_{L} \in \boldsymbol{F}_{L}(U) \times \boldsymbol{F}_{L}(U)\right)$ is called:

- reflexive if $R(x, x)=1$, for every $x \in U$;

- symmetric if $R(x, y)=R(y, x)$, for every $x, y \in U$;

- $\otimes$-transitive if $R(x, z) \otimes R(z, y) \leq R(x, y)$, for every $x, y, z \in U$.

If the $R$ is reflexive, symmetric and $\otimes$-transitive, then $R$ is $\otimes$-similarity.

Proposition 2.4 [7] Let $\boldsymbol{L}$ be a residuated lattice. If the $R \in \boldsymbol{F}_{L}(U) \times \boldsymbol{F}_{L}(U)$ is a reflexive and $\otimes$ transitive fuzzy relation, then for every $x, y \in U$

\section{Approximation operators in L-fuzzy approximation space}

Definition 3.1 [2] By an approximation space, we mean a triple $A=(U, I, k)$, where $U$ is a nonempty set called the universe, $I: U \rightarrow \boldsymbol{P}(U)$ is an uncertainty mapping, and $k: \boldsymbol{P}(U) \times \boldsymbol{P}(U) \rightarrow[0,1]$ is a rough inclusion function and satisfies: for every $X, Y \in \boldsymbol{P}(U)$

- $k(X, Y)=1$ if and only if $X \subseteq Y$;

- $k(X, Y)>0$ if and only if $X \cap Y \neq \emptyset$.

Definition 3.2 By a L-fuzzy approximation space, we mean a triple $\boldsymbol{A}=\left(U, F_{L}, K\right)$, where $U$ is a non-empty set called the universe, $F_{L}: U \rightarrow \boldsymbol{F}_{L}(U)$ is an uncertainty mapping, and $k: \boldsymbol{F}_{L}(U) \times \boldsymbol{F}_{L}(U) \rightarrow[0,1]$ is a rough inclusion function and satisfies: for every $X, Y \in \boldsymbol{F}_{L}(U)$

- $K(X, Y)=1$ if and only if $\wedge_{x \in U}[X(x) \rightarrow$ $Y(x)]=1$;

- $K(X, Y)>0$ if and only if $\vee_{x \in U}[X(x) \otimes$ $Y(x)]>0$.

The mapping $F$ may be viewed as a granulation function which assigned each $u \in U$ to a fuzzy set of $\mathbf{F}(U)$, i.e. an elementary granule of information. In this way an indexed family of fuzzy sets which is elementary granules of information from our perspective,

$$
F_{L}^{\rightarrow}(U)(x)=\bigvee_{u \in U}\left[F_{L}(u)\right](x)
$$

is obtained. Then $F_{L} \rightarrow(U)$ is a fuzzy semi-partition of $U$, i.e.

$$
\bigwedge_{x \in U} F_{L}^{\rightarrow}(U)(x)<1,
$$

or a fuzzy partition of $U$, i.e.

$$
\bigwedge_{x \in U} F_{L}^{\rightarrow}(U)(x)=1
$$

In the approximation space $\mathbf{A}$, we determine what fundamental properties any reasonable rough approximation mapping $f: \mathbf{F}_{L}(U) \rightarrow \mathbf{F}_{L}(U)$ should possibly posses. We distinguish two kinds of approximation mappings: lower and upper approximation mappings (in short low- and uppmappings). Such "rationality" postulates for lowand upp-mapping could have the following forms: $R(x, y)=\bigvee_{y \in U}(R(x, z) \otimes R(z, y))=\bigwedge_{y \in U}(R(x, z) \rightarrow R(z, y)) . \quad \begin{aligned} & \text { Every low-mapping } f \text { is decreasing. (i.e. for } \\ & \text { each } X \subseteq U, x \in U, f(X)(x) \leq X(x)) ;\end{aligned}$ 
- Every upp-mapping $f$ is increasing. (i.e. for each $X \subseteq U, x \in U, X(x) \leq f(X)(x)$ );

- If $f$ is a low-mapping, then for every $X \subseteq$ $U, f(X)(x)=1, K(f(x), X(x))=1$;

- If $f$ is an upp-mapping, then for every $X \subseteq$ $U, f(X)(x)=1, K(f(x), X(x))>0$;

- For each $X \subseteq U, f(X)$ is definable in $\mathbf{A}$;

- For each $X \subseteq U$ is definable in $\mathbf{A}, f(X)=X$.

In the L-fuzzy approximation space $\mathbf{A}=$ $\left(U, F_{L}, K\right)$, The mapping $F_{L}$ generates a L-fuzzy binary relation $R_{L} \in U \times U$, such that for every $x, y \in U$

$$
\left[F_{L}(x)\right](y)=R_{L}(x, y) .
$$

Then

- $R_{L}$ is reflexive if and only if for every $x, y \in U$, $\left[F_{L}(x)\right](x)=1$

- $R_{L}$ is symmetry if and only if for every $x, y \in$ $U,\left[F_{L}(x)\right](y)=\left[F_{L}(y)\right](x)$;

- $R_{L}$ is $\otimes$-transitive if and only if for every $x, y, z \in U,\left[F_{L}(x)\right](z) \otimes\left[F_{L}(z)\right](y) \leq$ $\left[F_{L}(x)\right](y)$;

- $R_{L}$ is $\otimes$-similarity if and only if for every $x, y, z \in U,\left[F_{L}(x)\right](z) \otimes\left[F_{L}(z)\right](y)=$ $\left[F_{L}(x)\right](y)$.

$X \subseteq \mathbf{F}(U)$ is definable in an approximation space $\mathbf{A}$ if and only if there is a set $Y \subseteq U$ such that

$$
X(x)=\bigvee_{y \in U}\left(\left(F_{L}(x)\right)(y) \otimes Y(y)\right)
$$

Let $\mathbf{C}=\{f(X) \mid f(x)$ is definable $\}$, then $\mathbf{C}$ is a L-fuzzy partition of the universe $U$.

For every $X \in \mathbf{F}_{L}(U), x \in U$, let

$$
\begin{aligned}
& {\left[D^{+}(x)\right](y)=R_{L}(y, x),} \\
& {\left[D^{-}(x)\right](y)=R_{L}(x, y) ;} \\
& {\left[D^{+}(X)\right](y)=\bigvee_{x \in X} R_{L}(y, x),} \\
& {\left[D^{-}(X)\right](y)=\bigvee_{x \in X} R_{L}(x, y) .}
\end{aligned}
$$

represent dominating set and dominated set with respect to $X$, respectively.

In this section, we consider some mappings $f_{1}, f_{2}, f_{3}, f_{4}: \mathbf{F}_{L}(U) \rightarrow \mathbf{F}_{L}(U)$, where for every
$X \in \mathbf{F}_{L}(U), x \in U$.

$$
\begin{aligned}
f_{1}(X)(x) & =\left(D^{+}(X) \otimes X\right)(x) \\
& =\bigvee_{y \in U}\left(R_{L}(y, x) \otimes X(y)\right), \\
f_{2}(X)(x) & =\left(D^{-}(X) \otimes X\right)(x) \\
& =\bigvee_{y \in U}\left(R_{L}(x, y) \otimes X(y)\right) ; \\
f_{3}(X)(x) & =D^{+}(X)(x) \otimes X(x) \\
& =\bigvee_{y \in U}\left(R_{L}(y, x) \otimes X(x)\right), \\
f_{4}(X)(x) & =D^{-}(X)(x) \otimes X(x) \\
& =\bigvee_{y \in U}\left(R_{L}(x, y) \otimes X(x)\right) .
\end{aligned}
$$

and their respective "dual" mappings $f_{1}^{d}, f_{2}^{d}, f_{3}^{d}, f_{4}^{d}$

$$
\begin{aligned}
f_{1}^{d}(X)(x) & =\left(D^{+}(X) \rightarrow X\right)(x) \\
& =\bigwedge_{y \in U}\left(R_{L}(y, x) \rightarrow X(y)\right), \\
f_{2}^{d}(X)(x) & =\left(D^{-}(X) \rightarrow X\right)(x) \\
& =\bigwedge_{y \in U}\left(R_{L}(x, y) \rightarrow X(y)\right) ; \\
f_{3}^{d}(X)(x) & =D^{+}(X)(x) \rightarrow X(x) \\
& =\bigwedge_{y \in U}\left(R_{L}(y, x) \rightarrow X(x)\right), \\
f_{4}^{d}(X)(x) & =D^{-}(X)(x) \rightarrow X(x) \\
& =\bigwedge_{y \in U}\left(R_{L}(x, y) \rightarrow X(x)\right) .
\end{aligned}
$$

If a set $X \in \mathbf{F}_{L}(U)$ is definable in an approximation space $\mathbf{A}$ if and only if there is $Y \in \mathbf{F}_{L}(U)$ such that for every $x \in U, X(x)=f_{0}(Y)(x)$.

Proposition 3.3 Consider any $f: \boldsymbol{F}_{L}(U) \rightarrow$ $\boldsymbol{F}_{L}(U) . \quad f(X)$ is definable for any $X \subseteq U$ if and only if there is a mapping $g: \boldsymbol{F}_{L}(U) \rightarrow \boldsymbol{F}_{L}(U)$ such that $f=f_{0} \circ g$.

Proposition 3.4 In the L-fuzzy approximation space $\boldsymbol{A}=\left(U, F_{L}, K\right)$ based on residuated lattice $\boldsymbol{L}$, it holds for every $X \in \boldsymbol{F}_{L}(U), x \in U$,

(1) If $R_{L}$ is symmetric, then $f_{1}(X)(x)=f_{2}(X)(x)$ and $f_{3}(X)(x)=f_{4}(X)(x)$;

(2) If $R_{L}$ is reflexive, then $f_{1}(X)(x)=f_{3}(X)(x)$ and $f_{2}(X)(x)=f_{4}(X)(x)$;

(3) If $R_{L}$ is $\otimes$-similarity, then $f_{1}(X)(x)=$ $f_{2}(X)(x)=f_{3}(X)(x)=f_{4}(X)(x)$ and $f_{i}(X)(x)=f_{i}(X)(x) \circ f_{i}(X)(x), i=1,2,3,4$; 
From the Proposition 3.3 and 3.4, we draw a conclusion

Corollary 3.5 Suppose that the L-fuzzy binary relation is $\otimes$-similarity. For any approximation operator $f$ in L-fuzzy approximation space $\boldsymbol{A}$, there exists a mapping $g: \boldsymbol{F}_{L}(U) \rightarrow \boldsymbol{F}_{L}(U)$ such that $f=f_{0} \circ g$

From the definition of L-fuzzy approximation operators $f_{i}, i=1,2,3,4$, the L-fuzzy approximation operators can represent other approximation operator by the compound of $f_{i}, i=1,2,3,4$. Such as the approximation operator of L-fuzzy rough set based on residuated lattice in [8], $f_{2}, f_{2}^{d}$ are upper and lower approximation operators respectively. In the approximation operator in [9], the upper and lower operators are represented as $f_{2}^{d} \circ f_{2}$ and $f_{2} \circ f_{2}^{d}$, respectively.

\section{Properties of L-fuzzy ap- proximation operators}

Proposition 4.1 For any sets $X, Y \subseteq \boldsymbol{F}_{L}(U)$, object $x, y \in U$, and $i=1,2,3,4$, it holds that:

(1) $f_{i}^{d} \leq i d \leq f_{i}^{d}$

(2) $f_{1}(x)$ is definable;

(3) $f_{i}\left(F^{\rightarrow}(U)\right)=f_{i}^{d}\left(F^{\rightarrow}(U)\right)=F^{\rightarrow}(U)$;

(4) $\left[f_{1}(x)\right](y)=1, K\left(\left[F_{L}(y)\right](x), X(x)\right)>0$;

(5) $\left[f_{1}^{d}(x)\right](y)=1, K\left(\left[F_{L}(y)\right](x), X(x)\right)=1$;

(6) $f_{i}(\emptyset)=\emptyset, f_{i}(U)=U$;

(7) $f_{i}$ and $f_{i}^{d}$ are monotone;

(8) $f_{i}(X \cup Y)=f_{i}(X) \cup f_{i}(Y)$;

(9) $f_{i}(X \cap Y) \subseteq f_{i}(X) \cap f_{i}(Y)$

(10) $f_{i}^{d}(X \cup Y) \supseteq f_{i}(X) \cup f_{i}(Y)$;

(11) $f_{i}^{d}(X \cap Y)=f_{i}^{d}(X) \cap f_{i}^{d}(Y)$.

IMTL-algebra is the special case of residuated lattice [11],[12] . The upper and lower approximation operators of L-fuzzy rough set based on IMTLalgebra are dual [13] . Similarity, in the L-fuzzy approximation space based on IMTL-algebra, the L-fuzzy approximation operator $f_{i}, i=1,2,3,4$ can find its dual operator.

Proposition 4.2 If the L-fuzzy approximation space $\boldsymbol{A}=\left(U, F_{L}, K\right)$ based on IMTL-algebra, the approximation operator $f_{i}, i=1,2,3,4$ have the their dual operator $f_{i}^{d}, i=1,2,3,4$, respectively. that is say for every $X \in \boldsymbol{F}_{L}(U), x \in U$,

$$
f_{i}(X)(x)=\sim f_{i}^{d}(\sim X)(x) .
$$

\section{Conclusions}

In approximation space $A$, the L-fuzzy approximation operators $f_{i}, i=1,2,3,4$ degenerate the classical approximation operators of generalized rough set as following:

(1) $f_{1}^{\prime}(X)=\bigcup\left\{R_{s}(x) \mid R_{s}(x) \cap X \neq \emptyset\right\}$, $f_{1}^{\prime d}(X)=\bigcap\left\{R_{s}(x) \mid R_{s}(x) \subseteq X\right\}$

(2) $f_{2}^{\prime}(X)=\bigcup\left\{R_{p}(x) \mid R_{p}(x) \cap X \neq \emptyset\right\}$, $f_{2}^{\prime d}(X)=\bigcap\left\{R_{p}(x) \mid R_{p}(x) \subseteq X\right\}$

(3) $f_{3}^{\prime}(X)=\left\{x \mid R_{s}(x) \cap X \neq \emptyset\right\}$, $f_{3}^{\prime d}(X)=\left\{x \mid R_{s}(x) \subseteq X\right\}$

(4) $f_{4}^{\prime}(X)=\left\{x \mid R_{p}(x) \cap X \neq \emptyset\right\}$, $f_{4}^{\prime d}(X)=\left\{x \mid R_{p}(x) \subseteq X\right\}$

where $R_{s}(x)$ and $R_{p}(x)$ are dominating set $R_{s}(x)=$ $\{y \mid y \in I(x)\}$ and dominated set $R_{p}(x)=\{y \mid x \in$ $I(y)\}$ with respect to $X$, respectively.

Following the methods [2], we can define the $f_{i}, i=5,6, \cdots$. But the properties of the approximation operators in L-fuzzy approximation space isn't thoroughly extended from the approximation in generalized approximation space in [2], such as, $f_{2}$ is L-fuzziness of $f_{0}^{\prime} \circ f_{1}^{\prime d}$, and those properties needs more researchers to study.

\section{Acknowledgements}

The authors are grateful to the referees for their valuable comments and suggestions. This work was supported by the National Natural Science Foundation of China (Grant No. 60474022) and the Research Fund for the Doctor Program of Higher Education (Grant No. 20060613007).

\section{References}

[1] Z. Pawlak, Rough sets. International Journal of Computer and Information Science, 11:341-356, 1982.

[2] A. Gomolińska, A Comparative Studey of Some Generalized Rough Approximations, Fundamenta Informaticae, 51:103-119, 2002.

[3] B. De Baets, R. Mesiar, $\mathcal{T}$-partitions, Fuzzy Sets and Systems 97:211-223, 1998.

[4] D. Dubois and H. Prade, Rough fuzzy sets and fuzzy rough sets, Internat. J. General Systems 17 (2-3):191-209, 1990.

[5] D. Dubois and H. Prade, Putting fuzzy sets and rough sets together, In Intelligent Decision Support, (Edited by R. Slowinski), Kluwer Academic, Dordrecht, 203-232, 1992. 
[6] N.N. Morsi and M.M. Yakout, Axiomatics for fuzzy rough sets, Fuzzy sets and Systems 100(13):327 342, 1998.

[7] A. M. Radzikowska, Etienne E. Kerre, A comparative study of fuzzy rough sets, Fuzzy Sets and Systems 126:137-155 2002.

[8] A. M. Radzikowska, Etienne E. Kerre, An algebraic characterization of fuzzy rough sets, Fuzzy Systems, 2004 IEEE International Conference, 109-114.

[9] T. Deng, Yanmei Chen, Wenli Xu, Qionghai Dai, A novel approach to fuzzy rough sets based on a fuzzy covering, Information Sciences 177:2308-2326, 2007.

[10] J. Pavelka, On fuzzy logic I: Many-valued rules of inference, II: Enriched residuated lattices and semantics of propositional calculi, III: Semantical completeness of some many-valued propositional calculi. Zeitschr. F. Math. Logik und Grundlagend. Math., 25: 45-52, 119-134, 447464, 1979.

[11] D. Pei, On equivalent forms of fuzzy logic systems NM and IMTL, Fuzzy Sets and Systems 138:187-195, 2003.

[12] F. Esteva, L. Godo, Monoidal t-norm-based logic: towards a logic for left-continuous tnorms, Fuzzy Sets and Systems 124:271-288, 2001.

[13] Z. Wu, K. Qin, L-Fuzzy Rough Set Based on Residuated Lattice and Its I-axiomatic and IIaxiomatic Approaches, Fuzzy Sets and Systems, to submit. 